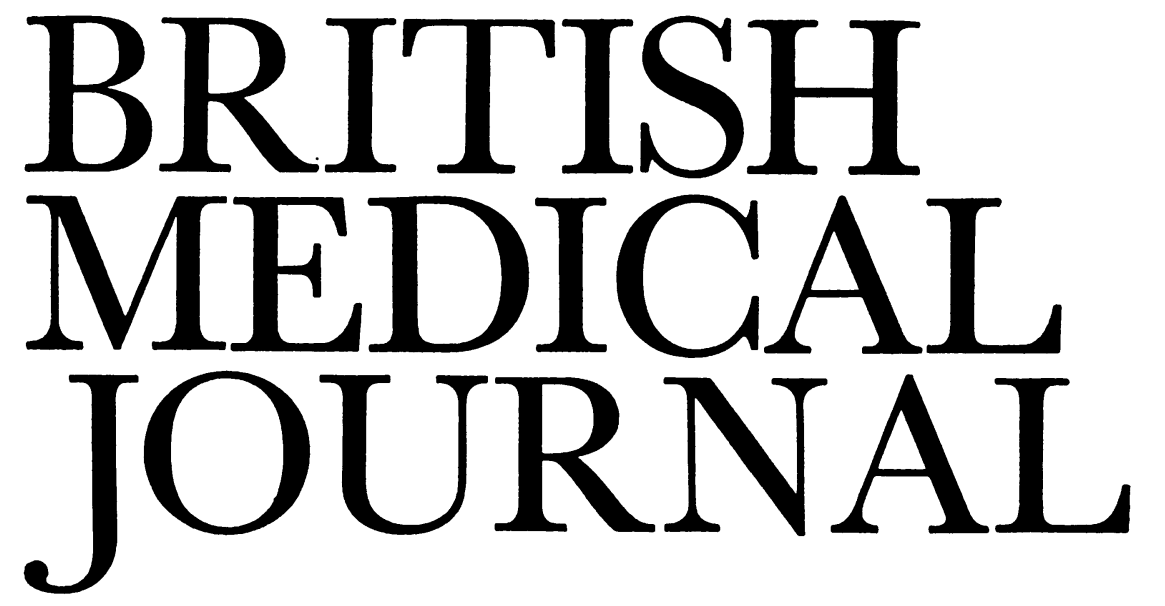

LONDON SATURDAY 1 APRIL 1972

\title{
When and Where Should Hypertension be Treated?
}

Since 1951 there has been conclusive evidence that features of severe hypertension, such as retinal haemorrhages and cotton-wool spots and left ventricular failure, can be rapidly reversed by treatment which lowers blood pressure. Treatment was soon shown to prolong life considerably in the malignant phase of hypertension, though many of these patients still die from renal failure.

During the 20 years that have passed since that time there has been a steady improvement in the drugs available and in the skill and organization which support their use. In consequence patients with milder and milder disease are being treated. Most of the published experience thus far in the long-term control of blood pressure has been gained from special clinics established in large regional hospitals. These clinics usually follow up their patients indefinitely, adjust the drug dosage, and carry out periodic investigations to evaluate cardiac and renal function. Special clinics of this kind have an esprit de corps of their own, and the patients who gravitate to them are often referred because of symptoms or complications-or the fear of them. Such individuals are particularly likely to be motivated to co-operate with treatment, and if there is also an efficient follow-up organization in the clinic the drop-out rate may be very low, so that the control of blood pressure over long periods is remarkably effective. Hypertension clinics have now been established in many countries. In the present issue of the B.M.F. (page 21) Professor K. L. Stuart and his colleagues from the University of the West Indies in Kingston, Jamaica, describe the successful operation of such a clinic in the control and follow-up of Jamaican patients with moderate and severe hypertension.

Hypertension clinics in hospitals cater for a tiny minority of all hypertensive patients, and if a deliberate effort is to be made to lower the blood pressure of all patients who ought to have it lowered for their benefit most will have to be treated by general practitioners. Who is eligible for treatment? Few control trials of antihypertensive treatment have been carried out. The most satisfactory were organized by the Veterans Administration in the United States. ${ }^{12}$ In the first Veterans study, a group of patients with a diastolic blood pressure between 115 and $129 \mathrm{~mm} \mathrm{Hg}$ were selected. The diastolic pressure of the patients on placebo averaged $118 \mathrm{~mm} \mathrm{Hg}$ while in the treated group it averaged $92 \mathrm{~mm}$
$\mathrm{Hg}$. There were four deaths and ten major complications in the placebo group and no deaths and no major complications in the actively treated group. Later, patients with diastolic pressures between 90 and $114 \mathrm{~mm} \mathrm{Hg}$ were studied. Nineteen related deaths and nine major complications occurred in the group on a placebo and eight deaths and no major complications in the treated group. The Veterans Administration group estimated that treatment was $75 \%$ effective in preventing deaths and major complications in patients with diastolic blood pressures between 105 and $114 \mathrm{~mm} \mathrm{Hg}$, but only $35 \%$ effective in a group with diastolic pressures between 90 and $104 \mathrm{~mm} \mathrm{Hg}$. All of the patients studied in both these investigations were middle-aged men. It is also noteworthy that nearly half the patients who fulfilled the blood pressure criteria for selection were eliminated from the trial because they attended irregularly or were unreliable in taking tablets. These results raise several important questions. Should all patients in the community with diastolic blood pressures above $90 \mathrm{~mm} \mathrm{Hg}$ be sought out by survey techniques and be offered treatment? Should all district general hospitals establish hypertension clinics to cater for the more severe cases among the enormous number of patients?

It would be wise not to extrapolate too far from the present evidence, but some tentative guidelines can be suggested. Men under 65 with diastolic blood pressures of $110 \mathrm{~mm} \mathrm{Hg}$ or higher should be offered antihypertensive treatment unless there is some strong reason to the contrary. Women in this age group should also be offered antihypertensive treatment, but the pressure level for selection might be set $5 \mathrm{~mm}$ higher. Most of these patients will be treated in general practice, but hospital clinics will be needed to help with difficult problems, and good follow-up is essential in either case. More evidence is needed about the effectiveness of treatment among middle-aged patients with diastolic pressures below $110 \mathrm{~mm} \mathrm{Hg}$, especially its role in the prevention of myocardial infarction. Present evidence suggests that reduction of blood pressure is most effective in preventing deaths from heart and renal failure and cerebral haemorrhage, and probably ineffective in preventing myocardial infarction. Organization of a clinical trial to evaluate treatment of patients with very mild hypertension would require very large numbers and poses difficult organizational problems. 
But the question is of substantial importance and should be answered.

The policy for elderly hypertensives is more difficult to define. There is little point in treating very old patients with hypertension with the aim of preventing a long-term risk. Yet hypertension becomes more frequent as age advances. Only $0.2 \%$ of the $30-39$-year-old age group have a diastolic pressure exceeding $110 \mathrm{~mm} \mathrm{Hg}$, but by the 60-69 decade $4.6 \%$ exceed this level. ${ }^{3}$ Some would argue strongly that antihypertensive treatment offers elderly people the best chance of reducing the risk of strokes and the consequent misery and disability that goes with them. Clinical trials are needed to determine how effective and practical such treatment would be in older people. ${ }^{3}$

1 Veterans Administration Co-operative Study Group on Antihypertensive Agents, Fournal of the American Medical Association, 1967 202, 1028.

2 Veterans Administration Co-operative Study Group on Antihypertensive Agents, Fournal of the American Medical Association, 1970, 213, 1143 .

3 World Health Organization, Technical Report Series No. 469. Geneva, 1971.

\section{Vagotomy for Duodenal Ulcer}

The operation of vagotomy for duodenal ulcer has been modified repeatedly over the past 20 years. Firstly, division of the vagal trunks at the oesophageal hiatus-truncal vagotomy-was practised. This denervates not only the stomach but also other abdominal viscera, and since denervation of the stomach leads to gastric stasis gastroenterostomy was added to overcome this unwelcome side effect. Pyloroplasty was then recommended instead of gastroenterostomy as being a more physiological operation and one which might prevent such unpleasant side effects as bilious vomiting and malabsorption due to poor food mixing.

Selective vagotomy was introduced because it seemed illogical to denervate most of the abdominal viscera just to reduce gastric acidity. Anterior selective vagotomy, preserving the hepatic branches of the anterior vagus, and posterior vagotomy, preserving the coeliac branches of the posterior vagus, were both tried. Finally, bilateral selective vagotomy became established, and though it lessened the incidence of postvagotomy diarrhoea it did not eliminate it. ${ }^{1}$ Selective vagotomy is now being superseded by a further refinement - denervation of only the acid-secreting cells of the stomach. This preserves the nerve supply of the antrum so that an additional drainage operation is claimed to be unnecessary. Variously called highly selective vagotomy, proximal gastric vagotomy, or parietal cell vagotomy, the initial results of this procedure have been mostly good, ${ }^{2} 3$ though there has been a recent adverse report on the clinical results. ${ }^{4} \mathrm{~A}$ note of caution was sounded by a study 5 which showed that there was a recovery of acid secretion over a period of 12 months after the operation. This may have been due to reinnervation, but as yet its clinical significance is uncertain.

As the nomenclature of vagotomy gets more complex so does the operation. A highly selective vagotomy takes longer to perform and in an obese patient may be a tedious and difficult procedure. Many surgeons with long operating lists and limited operating time will probably require definite evidence of the superiority of this new operation before they abandon their present practice. Recently some long-term results of vagotomy and pyloroplasty for duodenal ulcer have been compared ${ }^{6}$ with those obtained in a previous study ${ }^{7}$ of patients after truncal vagotomy and gastroenterostomy, truncal vagotomy and antrectomy, and partial gastrectomy. Surprisingly for any student of the surgical literature over the past 10 years, vagotomy and pyloroplasty proved to be the least satisfactory operation of the four. $\stackrel{c}{.}$ The proponents of vagotomy rightly claim that it is safer $\overrightarrow{\vec{F}}$ than partial gastrectomy, but the recurrent ulcer rate is $\frac{0}{9}$ higher and second operations for peptic ulcer are mcre $\frac{D}{O}$ difficult and dangerous. Therefore when the total surgical $\frac{\bar{N}}{\bar{N}}$ treatment required to control a patient's ulcer diathesis is considered the overall mortality rates may not be much of different.

The long-term sequelae of partial gastrectomy are well $\vec{\circ}$ documented and include weight loss, anaemia, malabsorption, bone disease, and pulmonary tuberculosis. Ten years $\stackrel{\circ}{\partial}$ ago it was taught that a history of pulmonary tuberculosis 3 . was a definite indication for vagotomy and drainage, but a i recent report ${ }^{8}$ of a fifteen-year follow-up of patients after ${ }_{0}^{\infty}$ vagotomy and gastroenterostomy shows this advice to be questionable. All the long-term metabolic effects previously $\vec{\rho}$ regarded as the result of gastric resection were observed, though they took longer to appear. An appreciable number (7\%) of patients developed pulmonary tuberculosis during the period of observation.

A high recurrent ulcer rate after vagotomy is almost always related to incomplete nerve section, and a recent study ${ }^{9}$ showed that surgeons varied greatly in their ability to do a complete vagotomy and that it was not always related to experience. Logically, therefore, surgeons should carry out insulin tests on their patients six months after $\mathbb{D}$ vagotomy, and if the incidence of incomplete vagotomy is high they might consider returning to gastric resection as their standard operation.

Most of the standard operations for peptic ulcer were introduced with enthusiasm, and their shortcomings became apparent only after a number of years. When the results of the various established procedures are compared objectively they are found to be very similar. ${ }^{7}$ An inevitable conclusion is that the patient and not the operation matters more. More attention has been directed towards the "gastric" component of the disease than to the "cerebral" component. A recent $\stackrel{9}{工}$ study showed that psychological testing before operation $N$ gave a fairly accurate prediction of those patients in whom $\frac{D}{O}$ a poor result was probable. ${ }^{10}$ Readers familiar with the Peter principle ${ }^{11}$ will recall that people advance in life until $\stackrel{N}{\circ}$ they reach a level at which they are incompetent. They sometimes resolve their dilemma by developing psychosomatic disease. For some patients a duodenal ulcer repre- $\stackrel{\varrho}{\subset}$ sents the pathology of success, and the chances of curing $\stackrel{\Phi}{\oplus}$ such an individual by operation are uncertain, to say the least.

1 Kennedy, T., and Connell, A. M., Lancet, 1969, 1, 899.

2 Amdrup, E., and Jensen, H. E., Gastroenterology 1970, 59, 522.

3 Johnston, D., British Medical fournal, 1972, 1, 179. Medical fournal, 1972, 1, 28.

${ }_{5}^{5}$ Amdrup, E., and Kragelund, E., Gut, 1971, 12, 866

${ }^{5}$ Amdrup, E., and Kragelund, E., Gut, 1971, 12, 866.

7 Goligher, J. C., et al., British Medical fournal, 1972, 1, 7.

8 Wheld, 1, 437.

9 Johnston, D., and Goligher, J. C., Gut, 1971, 12, 963

10 McColl, I., Drinkwater, J. E., Hulme-Moir, 1., and Donnan, S. P. B., Gut, $1971,12,856$.

11 Peter, L. J., and Hull, R., The Peter Principle. London, Souvenir Press Ltd., 1969. 